\title{
Expression of human FUS protein in Drosophila leads to progressive neurodegeneration
}

\author{
Yanbo Chen ${ }^{1,3^{*}}$, Mengxue Yang ${ }^{2 *}$, Jianwen Deng ${ }^{2}$, Xiaoping Chen ${ }^{3}, \mathrm{Ye} \mathrm{Ye}^{2}$, Li Zhu ${ }^{2}$, Jianghong Liu ${ }^{2}$, \\ Haihong $\mathrm{Ye}^{2}$, Yan Shen ${ }^{1}$, Yan $\mathrm{Li}^{2,3}$, Elizabeth J. Rao ${ }^{3,5}$, Kazuo Fushimi ${ }^{3}$, Xiaohong Zhou ${ }^{3}$, Eileen H. Bigio ${ }^{4}$,

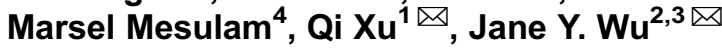 \\ ${ }^{1}$ National Laboratory of Medical Molecular Biology, Institute of Basic Medical Sciences, Chinese Academy of Medical Science \\ and Peking Union Medical College, Tsinghua University, Beijing 100730, China \\ 2 State Key Laboratory of Brain and Cognitive Science, Institute of Biophysics, Chinese Academy of Sciences, Beijing 100101, \\ China \\ ${ }^{3}$ Department of Neurology, Center for Genetic Medicine, Lurie Cancer Center, Northwestern University Feinberg School of \\ Medicine, 303 E. Superior St., Chicago, IL 60611 USA \\ ${ }^{4}$ The Cognitive Neurology \& Alzheimer's Disease Center, Northwestern University Feinberg School of Medicine, 303 E. Chicago \\ Ave., Chicago, IL 60611, USA \\ 5 Trumbull College, Yale University, New Haven, CT 06511, USA \\ $\triangle$ Correspondence: qixuangela@gmail.com (Qi Xu), jane-wu@northwestern.edu (J. Y. Wu) \\ Received June 17, 2011 Accepted June 23, 2011
}

\section{ABSTRACT}

Mutations in the Fused in sarcoma/Translated in liposarcoma gene (FUS/TLS, FUS) have been identified among patients with amyotrophic lateral sclerosis (ALS). FUS protein aggregation is a major pathological hallmark of FUS proteinopathy, a group of neurodegenerative diseases characterized by FUS-immunoreactive inclusion bodies. We prepared transgenic Drosophila expressing either the wild type (Wt) or ALS-mutant human FUS protein (hFUS) using the UAS-Gal4 system. When expressing Wt, R524S or P525L mutant FUS in photoreceptors, mushroom bodies (MBs) or motor neurons (MNs), transgenic flies show age-dependent progressive neural damages, including axonal loss in MB neurons, morphological changes and functional impairment in MNs. The transgenic flies expressing the hFUS gene recapitulate key features of FUS proteinopathy, representing the first stable animal model for this group of devastating diseases.

KEYWORDS frontotemporal lobar degeneration (FTLD), FUS proteinopathy, animal model, amyotrophic lateral sclerosis, neurodegeneration

\section{INTRODUCTION}

Since the landmark discovery of TAR-DNA binding protein of $43 \mathrm{kDa}$ (TDP-43) as a characteristic component of inclusion bodies in amyotrophic lateral sclerosis (ALS) and taunegative/ubiquitin-positive frontotemporal lobar degeneration (FTLD-U) (Neumann et al., 2006), a number of mutations have been identified in ALS patients in two genes encoding multi-functional RNA/DNA binding proteins, TDP-43 and FUS (reviewed in Neumann et al., 2006, 2009; Mackenzie et al., 2011). FUS-immunoreactive pathology has been reported in a range of neurodegenerative diseases, including ALS, FTLD$U$ and polyglutamine diseases. Approximately $10 \%$ of FTLD patients show FUS-immunoreactive pathology and are classified as FTLD-FUS (Neumann et al., 2009; Mackenzie et al., 2010). FUS mutations are recognized as the second most common cause of familial ALS in a Spanish population (Syriani et al., 2010).

The human FUS (also known as hnRNP P2) gene on chromosome 16 encodes a 526-amino acid RNA/DNA binding protein of versatile function, ranging from DNA repair, transcriptional regulation, splicing modulation, RNA transport to translational control (Crozat et al., 1993; Rabbitts et al., 1993; Prasad et al., 1994; Aman et al., 1996; Zinszner et al.,

"These authors contributed equally to the work. 
1997; Yang et al., 1998; Baechtold et al., 1999). FUS proteins are highly conserved during evolution, containing RNA recognition motif (RRM) and zinc finger domains (see peptide sequence alignment in supplementary Fig. S1).

FUS-deficient mice show defects in B cell development, chromosomal instability and perinatal lethality (Hicks et al., 2000). FUS-null neurons show developmental defects in dendritic spines (Fujii et al., 2005). However, no FUS inclusion body-like pathology has been reported in FUSdeficient mice. Thus far, no stable animal model for FUS proteinopathy has been published.

We generated and characterized transgenic flies expressing wild-type $(\mathrm{Wt})$ or ALS-mutant hFUS in various subpopulations of neurons. Such FUS transgenic flies show agedependent neurodegeneration with ALS-mutant FUS flies exhibiting more severe phenotype than those expressing the Wt FUS. These FUS transgenic flies recapitulate major clinical and pathological features of FUS proteinopathy, serving as a useful animal model for these debilitating neurodegenerative diseases.

\section{RESULTS}

To build an animal model for FUS proteinopathy, we used the Gal4-UAS system to express either Wt or ALS-associated R524S and P525L mutant hFUS as red fluorescent protein (RFP)-HA-tagged proteins. Flies expressing the RFP-HA vector alone were used as negative controls. Multiple transgenic fly lines were generated. Lines with comparable levels of expression were further characterized after crossing with different Gal4 drivers to express FUS in distinct neuronal subpopulations.

\section{Expression of Wt or ALS-mutant hFUS in fly eyes causes retinal degeneration}

FUS expression in fly photoreceptors was achieved using a GMR-Gal4 driver. Flies expressing either Wt or ALS-mutant hFUS exhibited a range of eye abnormalities. Different from transgenic flies expressing Wt TDP-43 that exhibited necrosis-like patches and ommatidial loss (Li et al., 2010), flies expressing Wt hFUS showed a reduction in the red eye pigment as compared with the controls, beginning at eclosion. No necrosis-like darkened areas were observed (Fig. 1). Ultrastructural abnormalities were detected by scanning electron microscopy (SEM) in hFUS-expressing fly eyes. Rough eye surfaces with disrupted ommatidia and bristle organization were observed in hFUS-expressing eyes (Fig. 2, panels $B 1-B 3$ to $E 1-E 3)$, whereas the control fly eyes remained normal (Fig. $2 \mathrm{~A} 1$ and 2A2). SEM at higher magnifications revealed ommatidial loss, ommatidial fusion, bristle loss or ectopic bristles and aberrant bristle budding in the inter-ommatidial spaces (Fig. 2B3-2E3). ALS-mutant FUS expressing eyes showed more severe ommatidial abnormalities. Furthermore, eye atrophy was observed in
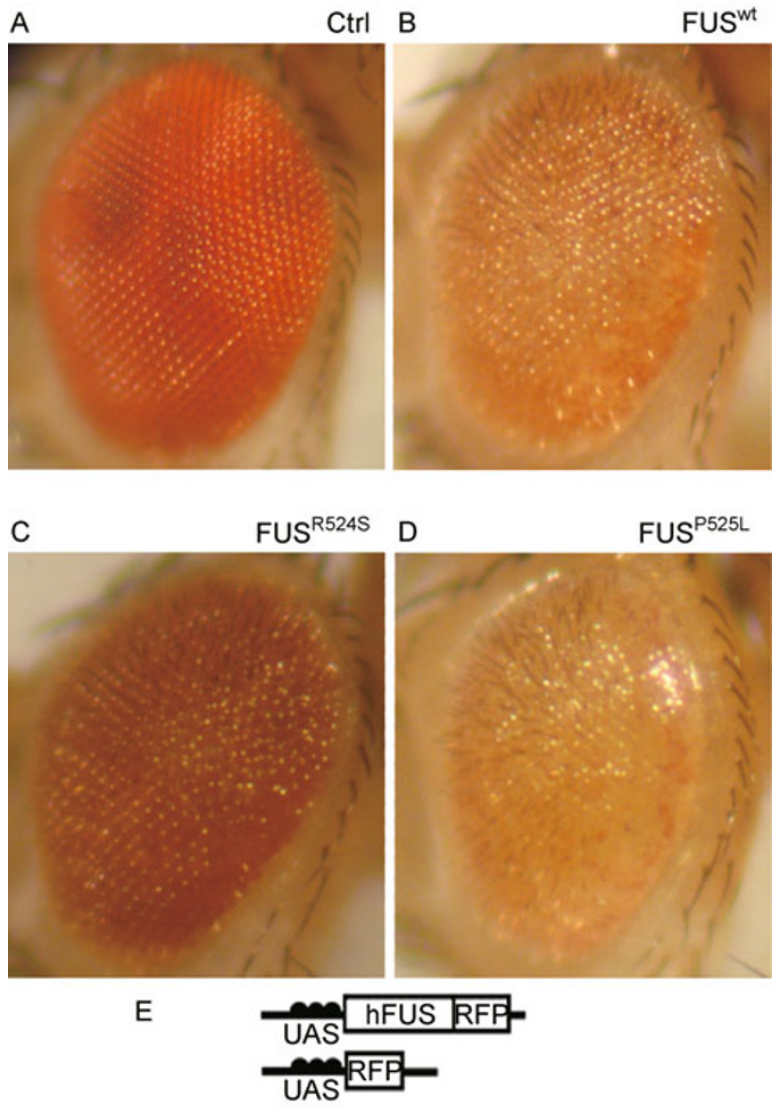

Figure 1. Light microscopic images of eyes from the control or Wt or ALS mutant FUS expressing transgenic flies (A-D) and a diagram illustrating the transgene constructs use (E).

two different lines expressing P525L mutant FUS (Fig. 2E1, for example). It was reported that expression of Gal4 protein might cause neurotoxicity and apoptosis in Drosophila (Kramer and Staveley, 2003; Rezával et al., 2007). However, our transgenic flies expressing RFP in photoreceptors showed no detectable changes in eyes. In addition, no neurodegeneration was observed in FUS transgenic flies without Gal4 expression. These data indicate that photoreceptor degeneration in hFUS expressing flies is not caused by nonspecific cytotoxicity associated with either RFP tag or Gal4 protein.

SEM imaging was also performed at different time points, day 5 and day 15 after eclosion (Fig. 3). Control RFP expressing eyes remained normal at day 15 . However, fly eyes expressing hFUS showed significant progression in degeneration by day 15 , with a marked increase in the rough eye area and more frequent findings of concave eye surfaces. Notably, in a line expressing the P525L mutant FUS, the eye surface appeared relatively normal at day 5 , but remarkably deteriorated by day 15 (Fig. 3D1 and 3D2). Only mild progression of rough eye phenotype, on the other hand, 


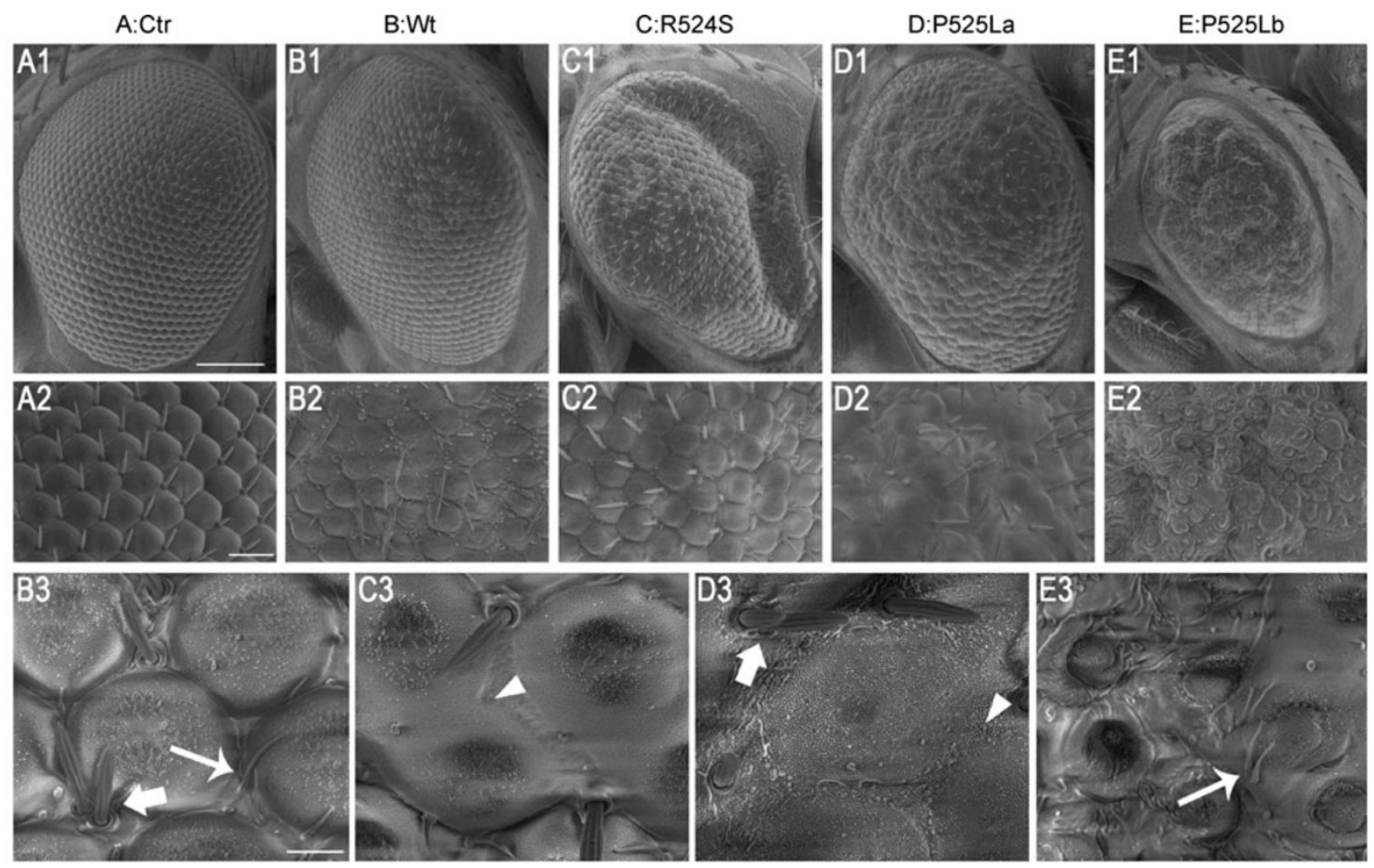

Figure 2. Expression of wild-type or ALS-mutant FUS in fly eyes leads to retinal degeneration as detected by scanning electron microscopy (SEM). (A-E) SEM images of fly eyes expressing red fluorescent protein (RFP) (Ctr) or Wt or mutant FUS. (A1 and A2) SEM images of fly eyes expressing RFP, showing normal morphology of ommatidia and inter-ommatidial bristles. (B-E) Flies expressing Wt (B), R524S (C) or P525L mutant FUS (E) show rough eyes (B1-E1), ectopic bristles and malformed ommatidia (B2-E2). Atrophic eyes were observed in a line expressing P525L FUS (E1). (B3-E3) Higher magnification images show detailed structural changes. Arrows mark ectopic bristles, with more than one bristles existing in a single inter-ommatidial space. Thin arrows indicate loss of normal bristles or aberrant budding of the inter-ommatidial bristles. Arrowheads mark fused ommatidia. Scale bar: A1-E1: $100 \mu \mathrm{m}$; A2-E2: $20 \mu \mathrm{m}$; B3-E3: $20 \mu \mathrm{m}$. All flies were raised at $25^{\circ} \mathrm{C}$, with at least 10 flies from each line examined by SEM. Genotypes: A1, A2: Ctr; GMR-Gal4/UAS-RFP; B1-B3: Wt, GMR-Gal4/UAS-Wt-FUS-RFP; C1-C3: R524S, GMR-Gal4/UASR524S-FUS-RFP; D1-D3 and E1-E3: two lines of P525L, GMR-Gal4/UAS-P525L-FUS-RFP.

was found in eyes expressing the Wt hFUS protein. Knocking down the endogenous Drosophila FUS homologue, cabeza, did not show detectable effects on the eye morphology.

\section{Expression of the Wt or ALS-mutant hFUS in MB neurons leads to progressive axonal loss}

To model FTLD-FUS, we investigated the effects of hFUS expression in the central nervous system by using the OK107-Gal4 driver and visualizing the MBs by a membrane-localized GFP in MB neurons (CD8::GFP, mGFP in the text). Z-stack images of MB lobes $\left(\alpha / \alpha^{\prime}, \beta / \beta^{\prime}\right.$, and $\gamma / \gamma^{\prime}$ lobes) formed by axon bundles of different subtypes of MB neurons were generated by confocal microscopy. Young (1day-old) flies expressing either Wt or mutant FUS showed decreased size of MBs and thinner lobes as compared with the control flies (Fig. 4). Similar to TDP-43 expressing flies ( $\mathrm{Li}$ et al., 2010), such axonal loss and lobe involvement were not symmetric, but often in a "group" fashion. That is, any of the lobes may be affected. However, once a particular lobe was involved, that lobe progressively degenerated. In many cases, one of the MBs may exhibit more degeneration than the MB on the opposite side.

\section{Expressing the Wt or ALS-mutant hFUS results in morphological changes of MNs}

We carefully examined the MNs expressing hFUS. Significant morphological changes in cell bodies and neuromuscular junctions (NMJ) were detected by fluorescent microscopy when OK371-Gal4 was used to drive the transgene expression in glutamatergic neurons of the ventral nerve cord (VNC) with neuronal morphology visualized by co-expression of mGFP. In control larvae, MNs in the VNC are assembled into well-organized clusters. Expression of hFUS led to disruption in MN clusters (Fig. 5B-D) with obvious cell body swelling in a fraction of MNs, especially in the posterior segment (arrowheads in corresponding panels of Fig. 5). To quantify the cell 
day 5
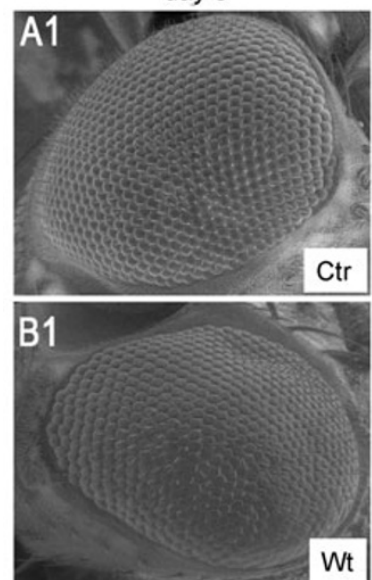

Wt
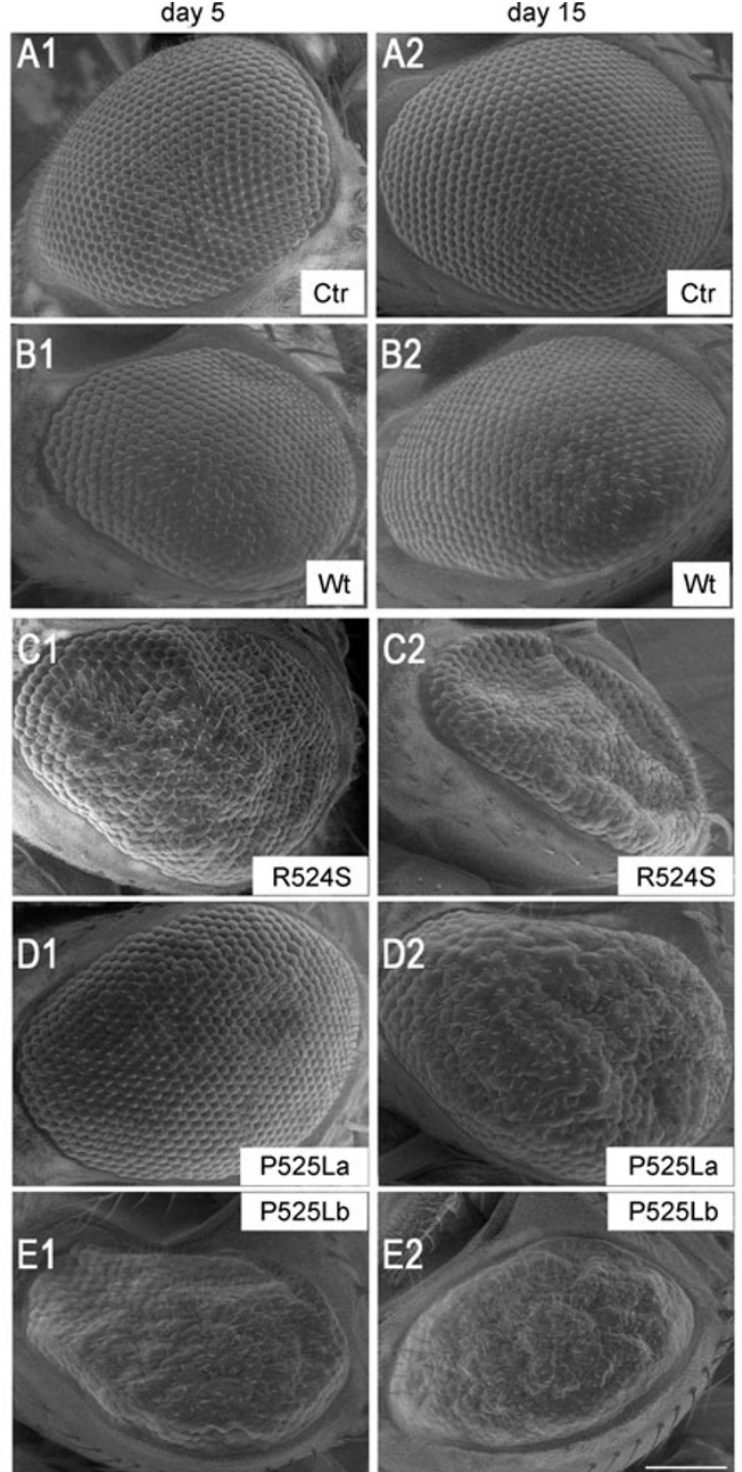

Figure 3. Progressive and age-dependent ultrastructural abnormalities in fly eyes expressing hFUS protein. Expression of Wt or mutant FUS driven by GMR-Gal4 leads to rough eye surfaces and disruption in the pattern of interommatidial bristles. (A) Flies expressing the RFP control show normal eye morphology. Organization of hexagonal ommatidia and interommatida bristles are not changed with the age in the control flies. (B) Fly eyes expressing Wt FUS show abnormal ommatida organization and shape as well as irregular appearance of the bristles, although no remarkable differences were observed at days 5 and 15 after eclosion. (C) Fusion of ommatidia was frequently observed in flies expressing R524S FUS, and the affected area became larger with aging. (D) In the P525La line expressing P525L hFUS, the eyes appeared to be almost normal by day 5 , whereas pervasive ommatidia fusion was discovered at day 15. (E) In another line, P525Lb, more severe retinal degeneration was detected with eye atrophy. $A$ few ommatidia were still recognizable at day 5 , but none was visible at day 15 . Scale bar: $100 \mu \mathrm{m}$. All flies were raised at $25^{\circ} \mathrm{C}$, and ten flies from each line were examined by SEM.

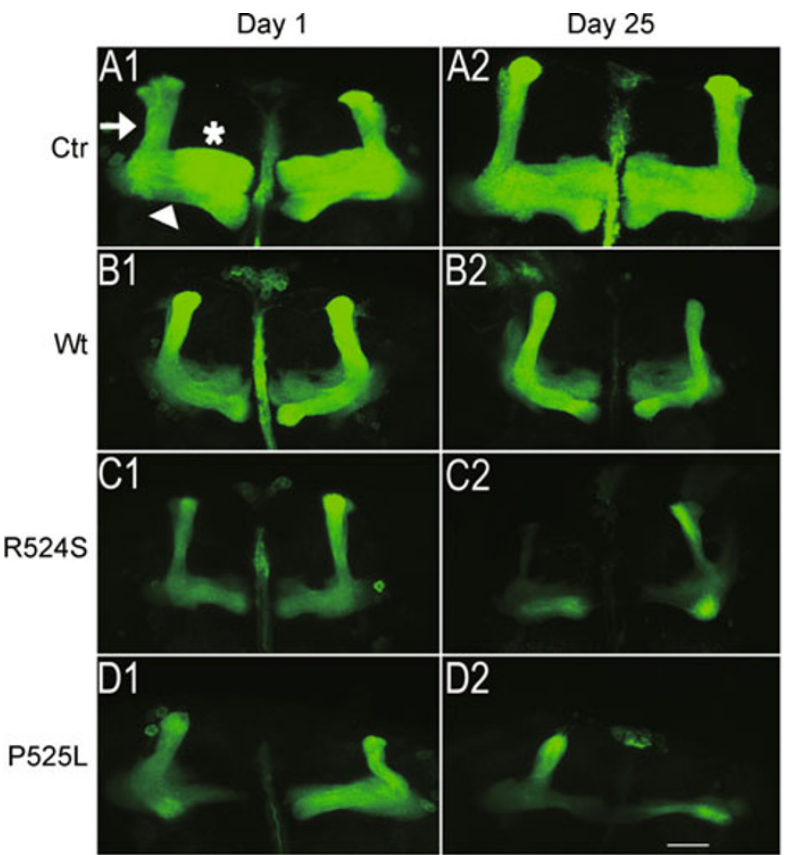

Figure 4. Expression of Wt or mutant FUS in mushroom body (MB) neurons leads to axonal degeneration. Axonal bundles of MB neurons are visualized by membrane GFP (CD8:: GFP). Confocal images were obtained in 20 Z-stacks to include all lobes in each brain and projected as single images. (A) Control flies (OK107-Gal4/UAS-RFP) showed normal MB lobe structures: $\alpha / \alpha^{\prime}$ (marked by the arrow), $\beta / \beta^{\prime}$ (arrowhead) and $\gamma / \gamma^{\prime}$ (star), at both day 1 and day 25. (B-D) Flies expressing Wt, R524S or P525L FUS (OK107-Gal4/UAS-Wt-FUS-RFP, OK107Gal4/UAS-R524S-FUS-RFP or OK107-Gal4/UAS-R524S-FUSRFP) showed a mild reduction in the size of all 3 lobes. Moreover, P525L FUS expressing flies showed a significant axonal loss by day 25 . Some MBs show asymmetric degeneration. For example, the right side MB in panel D2 shows more severe axonal loss. Scale bar: $20 \mu \mathrm{m}$.

swelling phenotype, we measured the cross-section areas of MN cell bodies. MNs expressing hFUS showed significant increases in $\mathrm{MN}$ cell body size as compared to controls $(p<0.001$; Fig. $5 \mathrm{E})$. The majority of the cell bodies were between 20 and $30 \mu \mathrm{m}^{2}$ in all groups. However, fly larvae expressing hFUS, either Wt, R524S or P525L, contained increased fractions of large MNs with cross section areas of $35 \mu^{2}$ or larger (Fig. 5F).

Furthermore, cytoplasmic FUS-RFP signals were detected in hFUS transgenic flies expressing ALS-mutant hFUS as revealed by biochemical fractionation experiments (data not shown) or by counterstaining MNs with a nuclear dye (Fig. 6). Low levels of cytoplasmic RFP signals were also detected in larvae expressing $\mathrm{Wt} \mathrm{hFUS}$.

MN axons project to their target muscles in each segment and form synapses with muscle cells within the varicosities termed as boutons. Two types of nerve processes were defined based on their morphology and the bouton sizes 

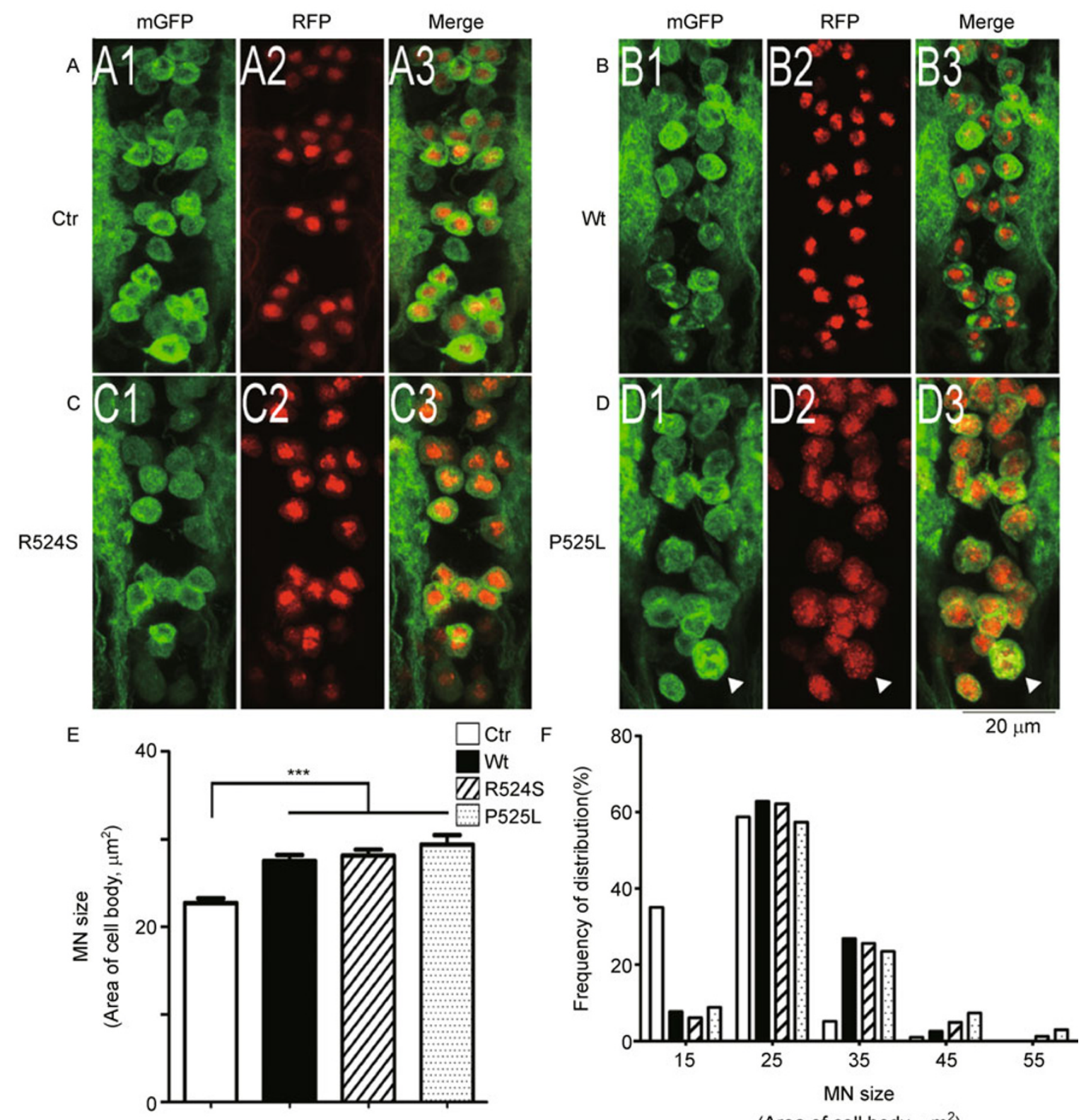

Figure 5. Morphological changes in motor neurons (MNs) of FUS transgenic flies. (A) In the ventral nerve cords (VNC) of the control larvae, the dorsal clusters were well organized. MNs are visualized by membrane localized GFP (mGFP). (B-D) The $3^{\text {rd }}$ instar larvae expressing Wt, R524S or P525L FUS show anomalous arrangement of motor neurons in dorsal clusters. Wt FUS protein is localized predominantly in the nucleus, with weak RFP signals detected in the cytoplasm. Remarkable cytoplasmically localized FUS protein is detected in MNs of both mutant groups with P525L showing stronger cytoplasmic signals in a punctate pattern. Some motor neurons in Wt or mutant FUS expressing larvae appear enlarged as compared to those in the control flies. An enlarged MN is indicated by arrowheads in panel D. Larval genotypes, in panel A: OK371-Gal4, mGFP/UAS-RFP; B: OK371-Gal4, mGFP/UAS-Wt-FUS-RFP; C: OK371-Gal4, mGFP/UAS-R524S-RFP; D: OK371-Gal4, mGFP/UAS-P525L-RFP. (E) Quantitative analysis of the projection area of motor neuron cell body. Wt, R524S or P525L FUS expressing larvae showed significant increase in cell size compared with control. More than 60 cells were measured in each group $\left(^{* * *}: p<0.001\right)$. (F) Frequency distribution of projection area of MN cell bodies. The cross-section areas of the majority of cells in all groups are between 20 and $30 \mu m^{2}$. Wt, R524S and P525L groups, however, exhibit increased frequencies of cells larger than $30 \mathrm{\mu m}^{2}$.

(Johansen et al., 1989). NMJs in the third instar larvae were revealed by fluorescent microscopy, with the short type I processes visualized and the total numbers of boutons in each axon terminal quantified (Fig. 7). A significant decrease 


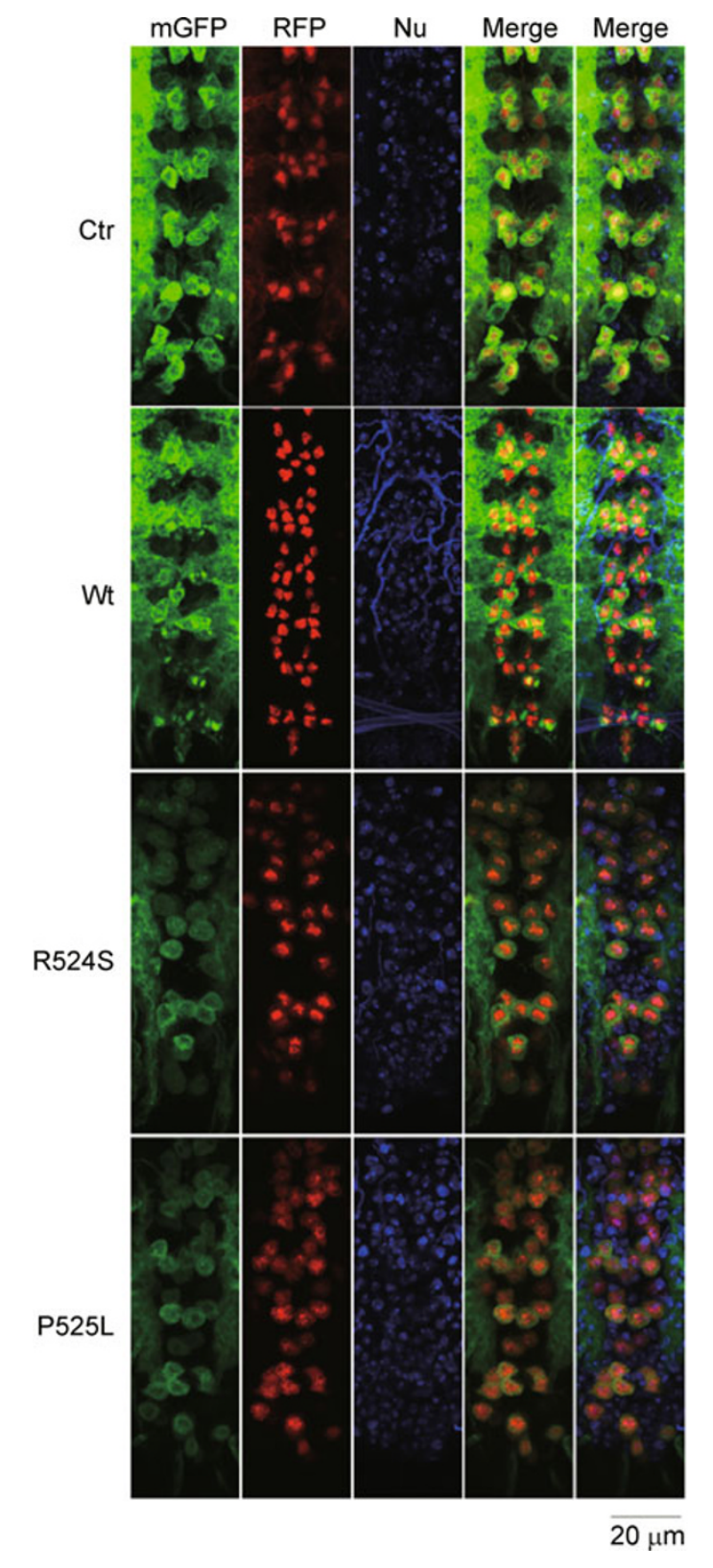

Figure 6. VNC motor neurons as counterstained with the Hoechst nuclear dye.

in the bouton number was detected in the hFUS-expressing flies, as compared to the controls (Fig. 7A-E). Remarkably, in flies expressing Wt hFUS that survived to the third instar, boutons were frequently enlarged as compared to the control larvae expressing RFP control ( $p<0.001$; Fig. 7B and 7F). The bouton size in P525L mutant FUS larvae was significantly reduced as compared to controls $(P<0.05$; Fig. 7D and $7 F$ ), although no significant difference was found in R524S mutant FUS expressing larvae $(p>0.05)$.

\section{Expression of hFUS in MNs impaires locomotive function of transgenic flies}

We examined the locomotive ability of the FUS transgenic flies. The larval movement in flies expressing hFUS either the Wt or ALS-mutant (R524S or P525L) was significantly reduced as compared with the controls (Fig. 7G). These FUS-expressing larvae moved more slowly with their tails lifted rather than anchored on the supporting surface, possibly as a result of paralysis of the posterior segments (Fig. $7 \mathrm{H}$ ). We surveyed multiple lines expressing ALS-mutant FUS. In larvae expressing R524S or P525L, (for example, in the fly line FUS-P525L2b that exhibited atrophic eyes), some showed such severe locomotion defects that they were unable to climb up the wall or hardly moving. There were no pupae hatched in most P525L mutant expressing fly lines tested, with one exception, in which the fly line survived to adulthood. This particular P525L mutant fly line remains to be further characterized to understand its differences from other lines. Overall, flies expressing R524S or P525L mutant show more severe $\mathrm{MN}$ damage and locomotive impairment. It should be noted that the mobility index in Fig. 7G was measured from those fly larvae that were able to move, therefore, representing an underestimate of motor impairment in R524S- or P525L-mutant FUS flies. No locomotive defects were observed in the flies in which FUS fly homolog cabeza was knocked down in MNs by RNA interference (RNAi).

\section{DISCUSSION}

FUS proteinopathy has recently emerged as a syndrome with shared neuropathological features but heterogeneous clinical manifestations. FUS proteinopathy may occur in patients with mutations in the coding region of the FUS gene or in patients without detectable FUS mutations (Neumann et al., 2009). The pathogenic mechanisms underlying FUS proteinopathy remain largely unknown, although it is clear that FUS proteinopathy not only affects motor neurons but also other neuronal populations such as cortical neurons. Data presented in this study provide strong evidence for a pathogenic role of hFUS and its ALS-associated mutants in neurodegeneration. Expression of either Wt or ALS-mutant FUS in different neuronal subpopulations, including photoreceptors, mushroom bodies and motor neurons, leads to agedependent progressive degeneration and functional deficits.

$\mathrm{MN}$ denervation is an important aspect in motor neuron diseases, including ALS (Jokic et al., 2006; Blijham et al., 2007). The presence of chromatolytic and swollen neurons is among the early pathological signs of MN degeneration in ALS (Okamoto et al., 1990; Sasaki and Maruyama, 1994). We systematically examined the effects of hFUS expression on the morphology and function of MNs in hFUS transgenic flies. Expression of two ALS-mutants, P525L and R524S, caused marked $\mathrm{MN}$ changes, including swollen cell bodies in ventral 


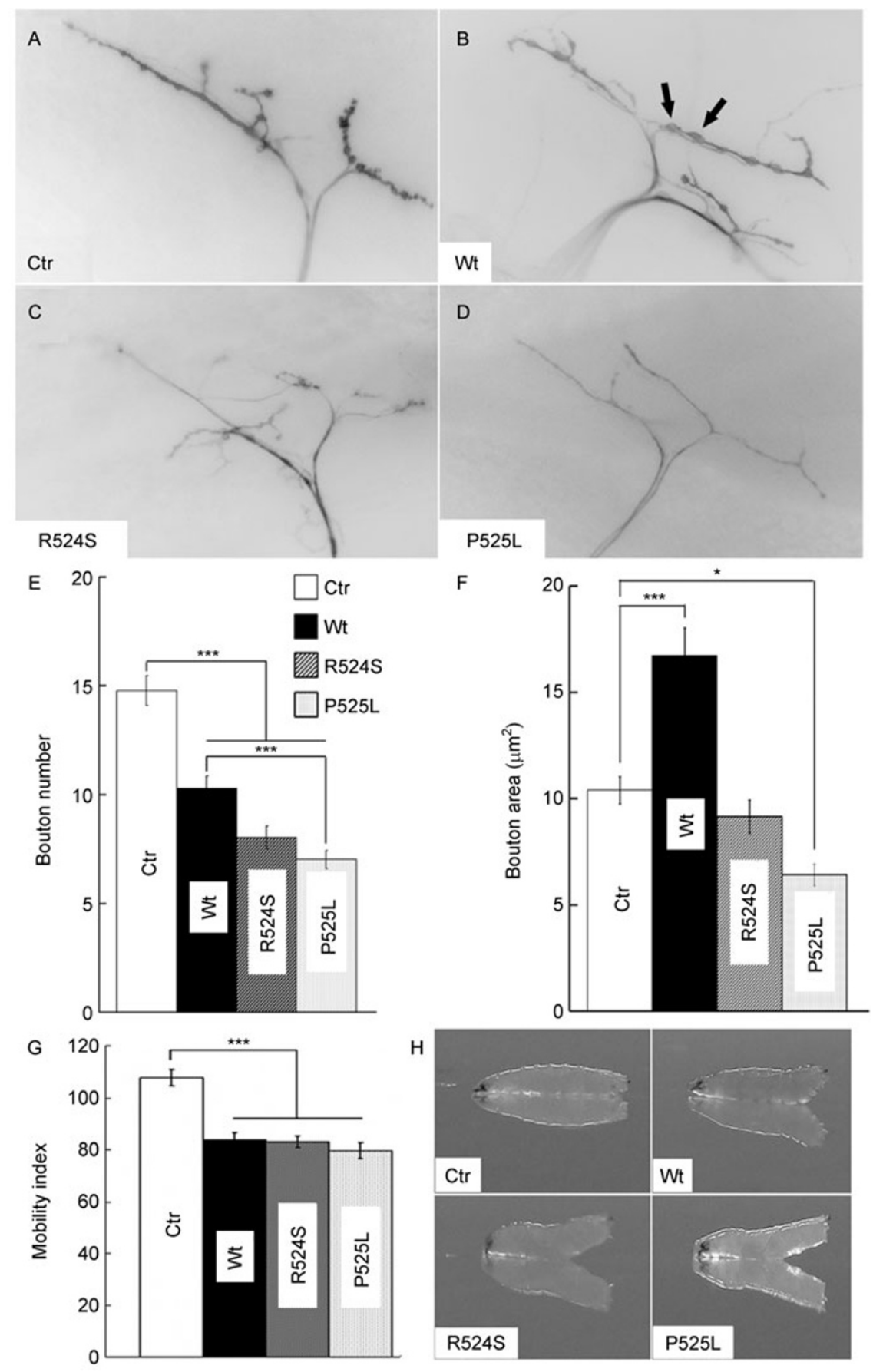

Figure 7. Morphological changes at the neuromuscular junctions (NMJs) and functional deficits in motor neurons (MNs) of FUS transgenic flies. (A-D) NMJ images from larvae expressing RFP vector control (Ctr) (A), Wt FUS (B), R524S FUS (C) or P525L FUS (D) in motor neurons. Numbers of type I bouton in each terminus were counted, and projected areas of boutons were measured. Enlarged boutons are marked by arrows in panel B. (E) Numbers of boutons per motor terminus were significantly reduced in FUS-expressing flies as compared to the control group (F). The projection areas of boutons in flies expressing Wt FUS were increased, whereas that in flies expressing P525L mutant FUS was decreased as compared to control flies. Genotypes of larvae in A: OK371-Gal4, mGFP/UAS-RFP; B: OK371-Gal4, mGFP/UAS-Wt-FUS-RFP; C: OK371-Gal4, mGFP/UAS-R524S-FUSRFP; D: OK371-Gal4, mGFP/UAS-P525L-FUS-RFP. (G) Locomotive deficits in flies expressing FUS in MNs. Third instar larvae expressing Wt or mutant FUS (R524S or P525L-line\#a) showed significant decrease in mobility index. More than 20 larvae were scored each group in each experiment. The data represent three independent experiments. All data were analyzed using one-way ANOVA $\left(*: p<0.05 ;^{* * *}: p<0.001\right)$. (H) The tail lifting phenotype observed in FUS expressing larvae. Control or FUS expressing larvae were crawling on an agar surface with their side view images taken. In the control group, larvae moved with their tails anchored on the surface, whereas FUS-expressing larvae lifted their tails while moving. 
nerve cords, and reduced NMJ boutons. FUS transgenic flies also show signs of motor denervation, with a significant reduction of mobility and viability of the larvae, mimicking clinical features of ALS. In the locomotion assay, the functional deficits were accompanied by tail paralysis, whereas the anterior body segment appeared relatively normal. This is consistent with the distal-to-proximal progression of motor neuron failure observed in ALS patients.

Although reduced numbers of $\mathrm{MN}$ boutons were found in flies expressing either the Wt or ALS-mutant FUS expressing larvae, enlarged boutons were frequently observed in flies expressing the Wt FUS. Interestingly, reductions in bouton numbers accompanied by bouton enlargement and increased active-zone density at the synapses have been reported in flies carrying metro or Fas II mutations (Stewart et al., 1996; Bachmann et al., 2010). Bouton enlargement has been proposed as a compensatory mechanism to increase functional active zones in the presence of motor denervation. In metro and Fas I/ mutants, such reciprocal correlation may result from an active redistribution of synaptic components to compensate for the reduction in bouton numbers, or alternatively, from a passive accumulation of continuously delivered synaptic materials. It remains to be investigated whether there is an increase in the active-zone density in the FUS transgenic flies. No obvious bouton enlargement was observed in larvae expressing ALS mutant FUS. This is possibly due to more rapid progression of neurodegeneration that exceeds the compensatory mechanism(s), and thus only residual varicosities could be detected in the 3rd instar larvae. Other possibilities include axonal transport impairment in MNs expressing mutant FUS, resulting in defects in bouton formation or axonal repair. It remains to be determined whether there was a transient bouton enlargement event in mutant FUS flies. Interestingly, mammalian FUS has been implicated in dendritic spine development (Belly et al., 2005; Fujii et al., 2005). FUS-null hippocampal pyramidal neurons showed abnormal spine morphology and reduced spine density (Fujii et al., 2005). The underlying mechanisms are not clear, though mRNA transport and local protein synthesis might be involved.

It is still an open question whether FUS proteinopathy is caused by haploinsufficiency (a loss of function of FUS gene products) or by gain-of-function neurotoxicity. In our study, down-regulating the cabeza gene, the Drosophila homolog of FUS, by RNAi in the eye or MNs, did not affect either the eye morphology or the larval locomotion. On the other hand, expression of Wt FUS was sufficient to produce the phenotypes similar to ALS-mutant FUS expressing lines, albeit less severe. This suggests that abnormal accumulation or decreased clearance of otherwise normal FUS protein product(s) could contribute to the pathogenesis of FUS proteinopathy, especially among patients without detectable FUS mutations. It will be interesting to investigate potential roles of both transcriptional and post-transcriptional mechanisms in the pathogenesis of FUS proteinopathy. It is possible that nucleotide sequence variations or mutations in the non-coding regions, including promoter, intronic or translational regulatory regions may contribute to both clinical and genetic heterogeneity of this group of neurodegenerative disorders, especially among patients without mutations in the coding region of the FUS gene.

Previous studies show mutant FUS protein is redistributed from the nucleus to the cytoplasm (Kwiatkowski et al., 2009; Vance et al., 2009). The C-terminal region of FUS is critical for its nuclear retention by interacting with Ran guanosine triphosphatase-dependent transport machinery (Dormann et al., 2010; Ito et al., 2010). Together, our data are consistent with the gain-of-toxicity hypothesis but do not exclude the possible involvement of impaired or loss of FUS function in the pathogenesis of the disease.

The two mutations studied in this report, R524S and P525L, are both located in the carboxyl terminal of the FUS protein (Fig. S1). Several reports of either sporadic or familial ALS patients show that patients with P525L mutation show very early onset and rapid disease progression (Chiò et al., 2009; Kwiatkowski et al., 2009; Bäumer et al., 2010; Huang et al., 2010). This mutation may contribute to juvenile ALS (Table S1). In contrast, clinical phenotypes associated with R524S mutation seem relatively milder with later onset and slower progression than that of P525L. Consistent with these observations, similar results were seen in our transgenic fly model, with flies expressing P525L mutant exhibiting more severe phenotypes as compared to ones expressing comparable levels of R524S mutant FUS protein.

The cytoplasmic sequestration of a normally shuttling nuclear protein and the formation of insoluble protein aggregates have been proposed as pathogenic mechanisms in TDP-43 proteinopathy, and formation of insoluble aggregates may count for this redistribution. Similar to TDP-43, FUS is a shuttling protein predominantly localized in the nucleus. Elevated levels of cytoplasmically localized FUS protein have been demonstrated by immunohistochemistry in postmortem ALS samples and in cell cultures. Although in cultured cells, only ALS-related mutations led to subcellular mislocalization, cytoplasmic FUS immunoreactivity was also detected in atypical FTLD-U (aFTLD-U) patients without detectable FUS gene mutations. FUS immunoreactivity has often been detected in basophilic inclusions in motor neuron diseases and aFTLD-U, which were tau-negative. It was noticed that a greater number of FUS-positive inclusion bodies (IBs) than ubiquitin- or p62-immunoreactive IBs were seen in both TDP-43 and FUS proteinopathies (Munoz et al., 2009; Fujita et al., 2010). This suggests that the formation of FUS containing basophilic inclusions is not necessarily ubiquitin-dependent. It remains unclear whether the protein mislocalization is a cause or consequence in these diseases. The molecular nature of the FUS gene products that are responsible for neurodegeneration in FUS proteinopathy 
needs to be determined in future studies.

Taken together, our transgenic Drosophila model recapitulates key clinical and pathological features of FUS proteinopathy, including ALS and FTLD-FUS. It is a powerful animal model for studying mechanisms of FUS proteinopathy and can be used in our future search for genetic modifiers or therapeutic agents that may alter clinical outcome of these diseases.

\section{MATERIALS AND METHODS}

\section{Transgenic constructs and fly strains}

The open reading frames encoding human FUS (the wild type, R524S or P525L mutants) were cloned in the pUAST vector, which contains a UAS sequence in the promoter region (Fig. 1) (Brand et al., 1993). FUS proteins, including the Wt or ALS mutants, contain the full-length FUS protein fused at its carboxyl terminus to RFP with a hemagglutinin (HA) tag. The sequences of the full length FUS cDNAs were verified. Transgenic flies expressing either RFP vector control or FUS-RFP protein were generated using a commercial fly injection service (Rainbow Transgenic Flies, Inc) in a W1118 background. The Cabeza-RNAi stock was obtained from Vienna Drosophila RNAi Center (VDRC). The Gal4 lines were obtained from Bloomington Stock Center.

\section{Fluorescent microscopy and scanning electron microscopy}

The third instar fly larvae or adult fly heads were dissected, and the brains were fixed with $4 \%$ paraformaldehyde (PFA) for $20 \mathrm{~min}$ at room temperature. After washing with PBS containing $0.1 \%$ Triton, the brains were counterstained with DAPI, and then mounted onto coverslips using mounting gel. Confocal images were taken under an Olympus FV1000 confocal microscope. Volumes of MBs were measured using Image-Pro Plus 7.0 (Media Cybernetics, Inc.). Images of NMJs were taken from the dorsal side of larvae under a Nikon Eclipse Ti fluorescent microscope.

For SEM, intact flies were progressively dehydrated in ethanol $(70 \%, 85 \%, 95 \%, 100 \%$; 60 min each step) and then in 100\% ethanol overnight. Dehydrated flies were applied to a CPD 030 critical point dryer, with $\mathrm{CO}_{2}$ as the drying agent. SEM images were acquired under the FEI Quanta 200 FEG electron microscope in the low vacuum mode.

\section{Larval movement assay}

The assay was done as described (Li et al., 2010). Briefly, the larval mobility index was measured as the number of peristaltic waves during the period of $2 \mathrm{~min}$ in the late third instar larvae expressing control RFP or FUS protein under the OK371-Gal4 driver in a controlled environment $\left(25^{\circ} \mathrm{C}\right.$, humidity $50 \% \pm 5 \%$, illumination $2800 \pm$ 100 lux).

\section{Statistical analysis}

All statistical analyses were performed using either one-way ANOVA, followed by Bonferroni multiple comparison for comparing individual groups. The bar graphs with error bars represent mean \pm standard error of the mean (SEM). Significance is indicated by asterisks: *: $p<0.05 ;{ }^{* *}: p<0.01{ }^{* * *}: p<0.001$.

\section{ACKNOWLEDGEMENTS}

We thank D. Kuo and other members of Wu group for critical reading of the manuscript. We thank members of the Wu lab for stimulating discussions and helpful suggestions. We are grateful for generous help from Drs. C. Wang, D. Han and J. Xie at National Center for Nanoscience and Technology in our SEM work. We thank D. Zhang for technical assistance in the early stage of the work. $\mathrm{JL}$ is supported by the National Basic Research Program (973 Program) (Grant No. 2009CB825402). YC, HY and QX are supported by the National Basic Research Program (973 Program) (Grant No. 2010CB529603).

Authors declare no competing financial interests.

Author contributions: EJR conceived the study. JYW and EJR designed the research experiments. YC, MY, JD, YY, XC, YL, XZ, KF, EJR and JYW performed the research and analyzed the data. $H Y, L Z$, $\mathrm{JL}, \mathrm{YS}, \mathrm{KF}, \mathrm{QX}$ and JYW supervised the experiments, discussed and analyzed the data. EHB and MM provided critical tissue samples and revised the manuscript. YC, MY, EJR and JYW wrote the paper.

It came to our attention that a study has just been published on line that reported similar neurodegeneration phenotypes in flies expressing FUS with other three ALS-mutations (Lanson et al., 2011).

\section{ABBREVIATIONS}

ALS, amyotrophic lateral sclerosis; FUS, Fused in sarcoma/Translated in liposarcoma gene; FTLD-FUS, frontotemporal lobar degeneration associated with FUS; FTLD-U, ubiquitin-positive frontotemporal lobar degeneration; Hfus, ALS-mutant human FUS protein; MBs, mushroom bodies; MNs, motor neurons; NMJ, neuromuscular junctions

Supplementary material is available in the online version of this article at http://dx.doi.org/10.1007/s13238-011-1065-7 and is accessible for authorized uers.

\section{REFERENCES}

Aman, P., Panagopoulos, I., Lassen, C., Fioretos, T., Mencinger, M., Toresson, H., Höglund, M., Forster, A., Rabbitts, T.H., Ron, D., et al. (1996). Expression patterns of the human sarcoma-associated genes FUS and EWS and the genomic structure of FUS. Genomics 37, 1-8.

Bachmann, A., Kobler, O., Kittel, R.J., Wichmann, C., Sierralta, J., Sigrist, S.J., Gundelfinger, E.D., Knust, E., and Thomas, U. (2010). A perisynaptic ménage à trois between Dlg, DLin-7, and Metro controls proper organization of Drosophila synaptic junctions. J Neurosci 30, 5811-5824.

Baechtold, H., Kuroda, M., Sok, J., Ron, D., Lopez, B.S., and Akhmedov, A.T. (1999). Human 75-kDa DNA-pairing protein is identical to the pro-oncoprotein TLS/FUS and is able to promote Dloop formation. J Biol Chem 274, 34337-34342.

Bäumer, D., Hilton, D., Paine, S.M., Turner, M.R., Lowe, J., Talbot, K., and Ansorge, O. (2010). Juvenile ALS with basophilic inclusions is a FUS proteinopathy with FUS mutations. Neurology 75, 611-618.

Belly, A., Moreau-Gachelin, F., Sadoul, R., and Goldberg, Y. (2005). Delocalization of the multifunctional RNA splicing factor TLS/FUS 
in hippocampal neurones: exclusion from the nucleus and accumulation in dendritic granules and spine heads. Neurosci Lett 379, 152-157.

Blijham, P.J., Schelhaas, H.J., Ter Laak, H.J., van Engelen, B.G., and Zwarts, M.J. (2007). Early diagnosis of ALS: the search for signs of denervation in clinically normal muscles. J Neurol Sci 263, 154-157.

Brand, A.H., and Perrimon, N. (1993). Targeted gene expression as a means of altering cell fates and generating dominant phenotypes. Development 118, 401-415.

Chiò, A., Restagno, G., Brunetti, M., Ossola, I., Calvo, A., Mora, G., Sabatelli, M., Monsurrò, M.R., Battistini, S., Mandrioli, J., et al., and the ITALSGEN Consortium. (2009). Two Italian kindreds with familial amyotrophic lateral sclerosis due to FUS mutation. Neurobiol Aging 30, 1272-1275.

Crozat, A., Aman, P., Mandahl, N., and Ron, D. (1993). Fusion of CHOP to a novel RNA-binding protein in human myxoid liposarcoma. Nature 363, 640-644.

Dormann, D., Rodde, R., Edbauer, D., Bentmann, E., Fischer, I., Hruscha, A., Than, M.E., Mackenzie, I.R., Capell, A., Schmid, B., et al. (2010). ALS-associated fused in sarcoma (FUS) mutations disrupt Transportin-mediated nuclear import. EMBO J 29, 2841-2857.

Fujii, R., Okabe, S., Urushido, T., Inoue, K., Yoshimura, A., Tachibana, T., Nishikawa, T., Hicks, G.G., and Takumi, T. (2005). The RNA binding protein TLS is translocated to dendritic spines by mGluR5 activation and regulates spine morphology. Curr Biol 15, 587-593.

Fujita, Y., Fujita, S., Takatama, M., Ikeda, M., and Okamoto, K. (2010). Numerous FUS-positive inclusions in an elderly woman with motor neuron disease. Neuropathology 31, 170-176.

Hicks, G.G., Singh, N., Nashabi, A., Mai, S., Bozek, G., Klewes, L., Arapovic, D., White, E.K., Koury, M.J., Oltz, E.M., et al. (2000). Fus deficiency in mice results in defective B-lymphocyte development and activation, high levels of chromosomal instability and perinatal death. Nat Genet 24, 175-179.

Huang, E.J., Zhang, J., Geser, F., Trojanowski, J.Q., Strober, J.B., Dickson, D.W., Brown, R.H. Jr, Shapiro, B.E., and Lomen-Hoerth, C. (2010). Extensive FUS-immunoreactive pathology in juvenile amyotrophic lateral sclerosis with basophilic inclusions. Brain Pathol 20, 1069-1076.

Ito, D., Seki, M., Tsunoda, Y., Uchiyama, H., and Suzuki, N. (2010). Nuclear transport impairment of amyotrophic lateral sclerosislinked mutations in FUS/TLS. Ann Neurol 69, 152-162.

Johansen, J., Halpern, M.E., Johansen, K.M., and Keshishian, H. (1989). Stereotypic morphology of glutamatergic synapses on identified muscle cells of Drosophila larvae. J Neurosci 9, 710-725.

Jokic, N., Gonzalez de Aguilar, J.L., Dimou, L., Lin, S., Fergani, A., Ruegg, M.A., Schwab, M.E., Dupuis, L., and Loeffler, J.P. (2006). The neurite outgrowth inhibitor Nogo-A promotes denervation in an amyotrophic lateral sclerosis model. EMBO Rep 7, 1162-1167.

Kramer, J.M., and Staveley, B.E. (2003). GAL4 causes developmental defects and apoptosis when expressed in the developing eye of Drosophila melanogaster. Genet Mol Res 2, 43- 47.

Kwiatkowski, T.J. Jr, Bosco, D.A., Leclerc, A.L., Tamrazian, E., Vanderburg, C.R., Russ, C., Davis, A., Gilchrist, J., Kasarskis, E.J., Munsat, T., et al. (2009). Mutations in the FUS/TLS gene on chromosome 16 cause familial amyotrophic lateral sclerosis. Science 323, 1205-1208.
Lanson, N.A. Jr, Maltare, A., King, H., Smith, R., Kim, J.H., Taylor, J. P., Lloyd, T.E. Pandey, U.B. (2011). A Drosophila model of FUSrelated neurodegeneration reveals genetic interaction between FUS and TDP-43. Hum Mol Genet 20, 2510-2523.

Li, Y., Ray, P., Rao, E.J., Shi, C., Guo, W., Chen, X., Woodruff, E.A. 3rd, Fushimi, K., and Wu, J.Y. (2010). A Drosophila model for TDP43 proteinopathy. Proc Natl Acad Sci U S A 107, 3169-3174.

Mackenzie, I.R., Munoz, D.G., Kusaka, H., Yokota, O., Ishihara, K., Roeber, S., Kretzschmar, H.A., Cairns, N.J., and Neumann, M. (2010). Distinct pathological subtypes of FTLD-FUS. Acta Neuropathol 121, 207-218.

Munoz, D.G., Neumann, M., Kusaka, H., Yokota, O., Ishihara, K., Terada, S., Kuroda, S., and Mackenzie, I.R. (2009). FUS pathology in basophilic inclusion body disease. Acta Neuropathol 118, 617-627.

Neumann, M., Rademakers, R., Roeber, S., Baker, M., Kretzschmar, H.A., and Mackenzie, I.R. (2009). A new subtype of frontotemporal lobar degeneration with FUS pathology. Brain 132, 2922-2931.

Neumann, M., Sampathu, D.M., Kwong, L.K., Truax, A.C., Micsenyi, M.C., Chou, T.T., Bruce, J., Schuck, T., Grossman, M., Clark, C.M., et al. (2006). Ubiquitinated TDP-43 in frontotemporal lobar degeneration and amyotrophic lateral sclerosis. Science 314 , 130-133.

Okamoto, K., Hirai, S., Shoji, M., Senoh, Y., and Yamazaki, T. (1990). Axonal swellings in the corticospinal tracts in amyotrophic lateral sclerosis. Acta Neuropathol 80, 222-226.

Prasad, D.D., Ouchida, M., Lee, L., Rao, V.N., and Reddy, E.S. (1994). TLS/FUS fusion domain of TLS/FUS-erg chimeric protein resulting from the $t(16 ; 21)$ chromosomal translocation in human myeloid leukemia functions as a transcriptional activation domain. Oncogene 9, 3717-3729.

Rabbitts, T.H., Forster, A., Larson, R., and Nathan, P. (1993). Fusion of the dominant negative transcription regulator $\mathrm{CHOP}$ with a novel gene FUS by translocation $\mathrm{t}(12 ; 16)$ in malignant liposarcoma. Nat Genet 4, 175-180.

Rezával, C., Werbajh, S., and Ceriani, M.F. (2007). Neuronal death in Drosophila triggered by GAL4 accumulation. Eur J Neurosci 25, 683-694.

Sasaki, S., and Maruyama, S. (1994). Decreased synaptophysin immunoreactivity of the anterior horns in motor neuron disease. Acta Neuropathol 87, 125-128.

Stewart, B.A., Schuster, C.M., Goodman, C.S., and Atwood, H.L. (1996). Homeostasis of synaptic transmission in Drosophila with genetically altered nerve terminal morphology. J Neurosci 16 , 3877-3886.

Syriani, E., Morales, M., and Gamez, J. (2010). FUS/TLS gene mutations are the second most frequent cause of familial ALS in the Spanish population. Amyotroph Lateral Scler 12, 118-123.

Vance, C., Rogelj, B., Hortobágyi, T., De Vos, K.J., Nishimura, A.L., Sreedharan, J., Hu, X., Smith, B., Ruddy, D., Wright, P., et al. (2009). Mutations in FUS, an RNA processing protein, cause familial amyotrophic lateral sclerosis type 6. Science 323 , 1208-1211.

Yang, L., Embree, L.J., Tsai, S., and Hickstein, D.D. (1998). Oncoprotein TLS interacts with serine-arginine proteins involved in RNA splicing. J Biol Chem 273, 27761-27764.

Zinszner, H., Sok, J., Immanuel, D., Yin, Y., and Ron, D. (1997). TLS (FUS) binds RNA in vivo and engages in nucleo-cytoplasmic shuttling. J Cell Sci 110, 1741-1750. 